\title{
TOTAL PARACOMPACTNESS OF REAL GO-SPACES
}

\author{
ZOLTAN BALOGH AND HAROLD BENNETT
}

(Communicated by Dennis Burke)

\begin{abstract}
A topological space is said to be totally paracompact (resp. totally metacompact) if every open base of it has a locally finite (resp. pointfinite) subcover. In this paper we characterize all totally paracompact GOspaces constructed on the real line. It turns out that in the class of GO-spaces on the real line, total paracompactness and total metacompactness are equivalent. Another consequence of our characterization is that totally metacompact GO-spaces on the real line are metrizable. Questions and partial results are given concerning total paracompactness in subspaces of real GO-spaces.
\end{abstract}

A topological space is said to be totally paracompact [Fo] (totally metacompact) if every base of it has a locally finite subcover (point-finite subcover). R. Telgarsky and H. Kok [TK] showed that the Michael Line [M] was not totally paracompact. In [Le] A. Lelek asked if the Sorgenfrey Line $[\mathbf{S}]$ was totally paracompact. This question was answered negatively by J. M. O'Farrell [OF $\mathbf{1}]$ using a technique that showed neither the Sorgenfrey Line nor the Michael Line is totally metacompact. Since both the Sorgenfrey Line and the Michael Line are real GO-spaces the following questions naturally arise:

1. What GO-spaces on the real line are totally metacompact or even totally paracompact?

2. Is total metacompactness equivalent to total paracompactness in real GOspaces?

In general the answer to Question 2 is no, since Heath's " $V$-space" $[\mathbf{H}]$ is totally metacompact and not (totally) paracompact.

In this paper it will be shown that total metacompactness and total paracompactness are equivalent in real GO-spaces. Moreover, we shall completely characterize all totally paracompact real GO-spaces in Theorem 2.3. From this characterization it follows that totally paracompact real GO-spaces are metrizable.

Recall that a linearly ordered topological space (=LOTS) is a linearly ordered set $X$ equipped with the usual open interval topology. If $\leq$ is the linear order on $X$ then a subset $C$ of $X$ is order convex if whenever $a$ and $b$ are in $C$ such that $a<b$, then $\{x \in X \mid a \leq x \leq b\} \subseteq C$. A generalized ordered space (=GO-space) is a linearly ordered set equipped with a $T_{1}$-topology for which there is a base consisting of convex sets. GO-spaces have been studied extensively (for example, see $\left[\mathbf{B L}_{\mathbf{1}}\right]$ or

Received by the editors December 10, 1985 and, in revised form, August 14, 1986. The results in this paper were presented at the University of Southwest Louisiana Spring Topology Conference in April of 1986. This conference was sponsored in part by N.S.F.

1980 Mathematics Subject Classification (1985 Revision). Primary 54F05, 54D18; Secondary $54 \mathrm{E} 35$.

Key words and phrases. Real GO-space, totally paracompact, totally metacompact, dense and codense example, metrizable. 
$\left[\mathbf{B L}_{\mathbf{2}}\right]$ ), but the fundamental paper is $[\mathbf{L u}]$. All undefined GO-space terminology will follow $[\mathbf{L u}]$.

A GO-space $X$ may be constructed on a LOTS $L$ by suitably altering the topology on $L$ so that $L$ is the pairwise disjoint union of subsets $R, E, I$, and $L$ where

$I=\{x \in X \mid\{x\}$ is open in $X\}$,

$R=\{x \in X-I \mid[x, \rightarrow)$ is open in $X\}$,

$L=\{x \in X-I \mid(\leftarrow, x]$ is open in $X\}$, and

$E=X-(R \cup L \cup I)$.

In this case $X$ is denoted by $\mathrm{GO}_{L}(R, E, I, L)$.

In this paper the interest is in GO-spaces constructed on the real line, that is, real GO-spaces. They will be denoted by $\mathrm{GO}(R, E, I, L)$.

If $(X, \tau)=\mathrm{GO}(R, E, I, L)$ then, as indicated, the GO-topology in $X$ is denoted by $\tau$. Let $\rho$ denote the usual topology in the real line. Thus if $\mathbf{R}$ and $\mathbf{Q}$ denote the real line and the set of rational numbers respectively, then the Michael Line is $\mathrm{GO}(\varnothing, \mathbf{Q}, \mathbf{R}-\mathbf{Q}, \varnothing)$ and the Sorgenfrey Line is $\operatorname{GO}(\mathbf{R}, \varnothing, \varnothing, \varnothing)$.

Let $\operatorname{cl}(A)$ always mean the $\rho$-closure of the set $A$ and let $|A|$ denote the cardinality of the set $A$. A $\rho$-perfect set $A$ is a nonvoid, $\rho$-closed set such that each point of $A$ is a $\rho$-limit point of $A$. A subset $A$ of $\mathbf{R}$ is said to be $\rho$-codense if $\mathbf{R}-A$ is $\rho$-dense. If $A \subseteq \mathbf{R}$, let $\tau \mid A$ denote the relative $\tau$-topology in the set $A$.

For the subspace $(A, \tau \mid A)$ we introduce the following notation.

$$
\begin{aligned}
i(A) & =\{x \in A:\{x\} \text { is open in } \tau \mid A\} ; \\
r(A) & =(R \cap A)-i(A) ; \\
l(A) & =(L \cap A)-i(A) ; \\
e(A) & =E \cap A .
\end{aligned}
$$

Notice that $A-e(A) \subseteq r(A) \cup l(A) \cup i(A)$.

\section{Dense and codense examples.}

DEFINITION 1.1. If $(\mathbf{R}, \tau)$ is a GO-space on the real line then a $\rho$-perfect subset $C$ of $\mathbf{R}$ is called a DCE (dense and codense example) if $i(C)$ is both $\rho$-dense and $\rho$-codense in $C$.

PROPOSITION 1.2. If $C$ is a $\rho$-perfect subset of $\mathbf{R}$ and $r(C)(l(C))$ is $\rho$-dense in $C$, then $C$ contains a $D C E$.

ProOF. Since $C$ is $\rho$-perfect, a $\rho$-nowhere dense, $\rho$-perfect subset $C_{1}$ of $C$ can be found such that all the endpoints of the open interval components of $\mathbf{R}-C_{1}$ are from $r(C)(l(C))$. This $C_{1}$ is the desired DCE.

If $C$ is a $\rho$-perfect subset of $\mathbf{R}$ let $C^{\prime}=\{x \in C \mid x$ is a two-sided limit point in $\rho \mid C\}$.

A DCE is of type I if $|i(C)|=\omega$ and of type II if $i(C) \cap C^{\prime}$ is $\rho$-dense in $C$.

PROPOSITION 1.3. If $C$ is a DCE then (' contains either a DCE of type I or a DCE of type II.

Proof. If $D=i(C) \cap C^{\prime}$ is $\rho$-dense in $C^{\prime}$ then $C$ is of type II. If $D$ is not $\rho$-dense in $C$, then there is an interval $[a, b]$ with $a$ and $b$ in $C^{\prime},|C \cap[a, b]|>\omega$, and $C \cap[a, b] \cap \operatorname{cl}(D)=\varnothing$. It follows that $C_{1}=C \cap[a, b]$ is a DCE of type I.

DEFINITION 1.4. A closed interval $[a, b]$ is nice if $a$ and $b$ are in $i(C)$ and $|[a, b] \cap C|>\omega$. A closed interval $[a, b]$ is normal if both $a$ and $b$ are in $i(C) \cap C^{\prime}$. 


\section{LEMMA 1.5. A DCE $C$ of type I is not totally metacompact.}

ProOF. Given a nice interval $P=[a, b]$, construct an infinite disjoint family $\tau(P)$ of nice intervals and a family $\mathcal{G}(P)$ of $\tau \mid C$-open sets in the following fashion: First pick an arbitrary point $e \in i(C) \cap(a, b)$. Then $e$ is a $\rho$-limit point of either $(a, e) \cap C$ or $(e, b) \cap C$. Without loss of generality let $e$ be a $\rho$-limit point of $(a, e) \cap C$. Since $i(C)$ is dense in $C$ there is an infinite disjoint family $\tau(P)$ of nice subintervals of $(a, e)$ converging to $e$ in $\rho \mid C$ (i.e., each open interval containing $e$ contains all but finitely many members of $\tau(P))$. Without loss of generality the members of $\tau(P)$ may be assumed to have diameter less than $(b-a) \cdot 2^{-1}$.

If $x \in(\bigcup \tau(P))-i(C)$, let $Q=[c, d]$ be the unique element in $\tau(P)$ containing $x$. Let

$$
G(P, x)= \begin{cases}((c, x] \cap C) \cup\{a\} & \text { if } x \in l(C), \\ ([x, d) \cap C) \cup\{b\} & \text { if } x \in r(C), \\ ((c, d) \cap C) \cup\{a, b\} & \text { if } x \in e(C) .\end{cases}
$$

In any case $G(P, x)$ is a $\tau \mid C$-open set. Let

$$
\mathcal{G}(P)=\{G(P, x) \mid x \in \bigcup \tau(P)-i(C)\} .
$$

A sequence $\{P(n) \mid n \in \omega\}$ of infinite disjoint families of nice intervals can be defined inductively by letting $P(0)=\{[a, b]\}$ consist of a nice interval and, for each $n \in \omega$, let $P(n+1)=\bigcup\{\tau(P): P \in P(n)\}$. Further, for every $n \in \omega$, let $\mathcal{G}(n)=\bigcup\{\mathcal{G}(P): P \in P(n)\}$, and let $\mathcal{G}=\bigcup\{\mathcal{G}(n): n \in \omega\}$.

Since in the definition of $\tau(P), e$ was a $\tau \mid C$-isolated point, it easily follows that $\bigcup \mathcal{P}(n)$ is $\tau \mid C$-closed for each $n \in \omega$. Thus the set $F=\bigcap\{(\bigcup \mathcal{P}(n)) \cap C \mid n \in \omega\}$ is $\tau \mid C$-closed. Let $\not{H}$ be a base for the points of $C-F$ such that no member of $\not{H}$ intersects $F$. It is easily seen that $B=\not \forall \cup \mathcal{G} \cup\{\{x\} \mid x \in i(C)\}$ is a base for $\tau \mid C$.

Suppose $B$ has a point-finite subcover $B^{\prime}$. Let $\mathcal{G}(n)^{\prime}=B^{\prime} \cap \mathcal{G}(n)$ for each $n \in \omega$. Since each $G(P, x)$ contains at least one of the endpoints of $P$ and $\mathcal{G}(n)^{\prime}$ is pointfinite, for each $n \in \omega$ and $P \in P(n)$ there are disjoint members $Q(P, 0)$ and $Q(P, 1)$ in $P(n+1)$ such that $Q(P, 0) \cup Q(P, 1) \subseteq P$ and $\left(\cup \mathcal{G}(n)^{\prime}\right) \cap(Q(P, 0) \cup Q(P, 1))=\varnothing$.

For each $f \in{ }^{\omega_{2}} 2$ define a monotone decreasing sequence of nice intervals as follows: Let $K(f, 0)=[a, b], K(f, 1)=Q([a, b], f(1))$, and if $K(f, i)$ is defined for $i \leq n$ let $K(f, n+1)=Q(K(f, n), f(n+1))$. For each $f \in{ }^{\omega} 2$ let $\{x(f)\}=$ $\bigcap\{K(f, n) \cap C \mid n \in \omega\}$. Notice that $F_{1}=\left\{x(f) \mid f \in \omega^{\omega}\right\} \subseteq F$. Since $\left|F_{1}\right|>\omega$ and $|i(C)|=\omega$, there exists $x(f) \in F_{1}-i(C)$. For each $n \in \omega, x(f) \notin \bigcup \mathcal{G}(n)^{\prime}$. Thus, since $x(f) \notin i(C)$ and $x(f) \notin \bigcup \not{H}$, no member of $B^{\prime}$ contains $x(f)$. From this contradiction it follows that $B$ has no point-finite subcover.

\section{LEMMA 1.6. A DCE of type II is not totally metacompact.}

ProOF. Given a normal interval $P=[a, b]$ an infinite family $\tau(P)$ of normal intervals and a family $\mathcal{G}(P)$ of $\tau \mid C$-open subsets can be constructed as follows. Let $E=\left\{t_{1}, t_{2}, \ldots\right\}$ be a countable $\rho$-dense subset of $\left(i(C) \cap C^{\prime}\right) \cap[a, b]$. Pick a decreasing sequence $\left\{x_{i} \mid i \in \omega\right\}$ of elements of $E$ that converges to $a$ and an increasing sequence $\left\{y_{i} \mid i \in \omega\right\}$ of elements of $E$ that converges to $b$ such that $x_{0}<y_{0}$. Since $a$ and $b$ are in $C^{\prime}$ and $D=i(C) \cap C^{\prime}$ is $\rho$-dense in $C$ such sequences exist. 
Let

$$
\tau(P)=\left\{\left[x_{n+1}, x_{n}\right] \mid n \in \omega\right\} \cup\left\{\left[y_{n}, y_{n+1}\right] \mid n \in \omega\right\} \cup\left\{\left[x_{0}, y_{0}\right]\right\} .
$$

Clearly $\bigcup T(P)=(a, b)$.

If $x \in P \cap(C-i(C))$, then a $\tau \mid C$-open set $G(P, x)$ is defined in the following fashion. Let $[c, d]$ be the unique member of $\tau(P)$ such that $x \in(c, d)$. Let

$$
G(P, x)=([x, d) \cap C) \cup([x, b] \cap i(C))
$$

if $x \in r(C)$, or $x \in e(C)$ and $x$ is the right endpoint of an interval component of $\mathbf{R}-C$. Let

$$
G(P, x)=((c, x] \cap C) \cup([a, x] \cap i(C))
$$

if $x \in l(C)$ or $x \in e(C)$ and $x$ is the left endpoint of an interval component of $\mathbf{R}-C$. Finally, let

$$
G(P, x)=((c, d) \cap C) \cup([a, b] \cap i(C)),
$$

if $x$ is none of the above.

Next define a sequence $\{\tau(n) \mid n \in \omega\}$ of families of normal intervals in the following way. Let $\tau(0)$ consist of a single normal interval $P_{0}$. If $\tau(0), \tau(1), \ldots, \tau(k)$ are defined let $\tau(k+1)=\bigcup\{\tau(P) \mid P \in T(k)\}$ and make sure $t_{k+1}$ is one of the endpoints of some interval in $\tau(k+1)$. For each $n \in \omega$ let

$$
\mathcal{G}(n)=\{G(P, x) \mid P \in \tau(n), x \in P \cap(C-i(C))\} .
$$

Since $\left\{t_{1}, t_{2}, \ldots, t_{n}\right\}$ is used in defining $\mathcal{G}(n)$ it follows that $\mathcal{G}=\bigcup\{G(n) \mid n \in \omega\}$ is a base for points in $P_{0} \cap(C-i(C))$. Thus $B=\mathcal{G} \cup\left\{\{x\} \mid x \in P_{0} \cap i(C)\right\}$ is a base for $\tau \mid P_{0} \cap C$.

Suppose $B^{\prime}$ is a point-finite subcover of $B$. Let $\mathcal{G}(n)^{\prime}=\mathcal{G}(n) \cap B^{\prime}$ for each $n \in \omega$ and let $\mathcal{G}^{\prime}=\mathcal{G} \cap B^{\prime}$.

The following definition is convenient. A set $P \in \tau(n+1)$ is $n$-semicovered if, for every $i \leq n,\left(\bigcup \mathcal{G}(i)^{\prime}\right) \cap(P-i(C))=\varnothing$ and $\bigcup \mathcal{G}(n)^{\prime} \supseteq P \cap i(C)$.

Claim 1. If $P \in \tau(n+1)$ is $n$-semicovered, then there is a $j>n$ and $Q \in T(j+1)$ such that $Q \subseteq P$ and $Q$ is $j$-semicovered.

To see that Claim 1 is true let $j$ be the first integer greater than $n$ such that $\left(\bigcup \mathcal{G}(j)^{\prime}\right) \cap(P-i(C)) \neq \varnothing$. Then there exists $P_{1} \in \tau(j)$ such that $P_{1} \subseteq P$ and $\left(\bigcup \mathcal{G}(j)^{\prime}\right) \cap\left(P_{1}-i(C)\right) \neq \varnothing$. If $G \in \mathcal{G}(j)^{\prime}$ and $G \cap\left(P_{1}-i(C)\right) \neq \varnothing$, then $G$ contains one of the endpoints of $P_{1}$. Since $\mathcal{G}(j)^{\prime}$ is point-finite it follows that $\mathcal{G}\left(P_{1}\right)^{\prime}=\{G \in$ $\left.\mathcal{G}(j)^{\prime} \mid G \cap\left(P_{1}-i(C)\right) \neq \varnothing\right\}$ is a finite set. Thus $\bigcup\left(\mathcal{G}(j)^{\prime}\right) \cap(Q-i(C))=\varnothing$ for all but finitely many $Q \in \tau\left(P_{1}\right)$. On the other hand, any $G \in \mathcal{G}\left(P_{1}\right)^{\prime}$ covers all isolated points (i.e., elements of $i(C)$ ) of infinitely many $Q \in \tau\left(P_{1}\right)$. Hence there is a $j$-semicovered $Q \subseteq P_{1} \subseteq P$ with $Q \in \tau\left(P_{1}\right) \subseteq P(j+1)$.

Claim 2. There is an $n \in \omega$ and a $P \in T(n+1)$ such that $P$ is $n$-semicovered.

The proof of Claim 2 is similar to the proof of Claim 1 and will be omitted.

By Claims 1 and 2 there is a sequence $n_{0}<n_{1}<\cdots$ of natural numbers and a corresponding nested sequence of intervals $P_{1}, P_{2}, \ldots$ such that for each $j \in \omega$, $P_{j} \in T\left(n_{j}+1\right)$ and $P_{j}$ is $n_{j}$-semicovered. Let $x \in \bigcap\left\{C \cap P_{j} \mid j \in \omega\right\}$. If $x \in C-i(C)$ then, from the definition of $n_{j}$-semicovered, it follows that $x \notin \bigcup \mathcal{G}^{\prime}$. Thus $B^{\prime}$ does not cover $x$. If $x \in i(C)$ then, from the definition of $n_{j}$-semicovered, $\mathcal{G}^{\prime}$ is not pointfinite at $x$. Thus $P_{0} \cap C$ is not totally metacompact. Since total metacompactness is a closed hereditary property it follows that $C$ is not totally metacompact.

By Proposition 1.3 and Lemmas 1.5 and 1.6 the following theorem is established. 
THEOREM 1.7. No DCE is totally metacompact.

2. The main theorem. To obtain the characterization of totally paracompact real GO-spaces, the following two lemmas are used.

LEMMA 2.1 (R. TELGARSKY $[\mathbf{T}]$ ). If a space $X$ is paracompact and locally totally paracompact, then it is totally paracompact.

LEMMA 2.2. If a space $X$ has a compact subset $C$ such that the subspace $X-C$ is totally paracompact, then $X$ is totally paracompact.

The proof of Lemma 2.2 is left to the reader. Also note that if $X$ is a GO-space on the real line, then in Lemma 2.2 , it is enough to assume that $X-C$ is locally totally paracompact. Indeed, since GO-spaces on the real line are hereditarily paracompact $[\mathbf{L u}]$, Lemma 2.1 applies to show that $X-C$ is totally paracompact.

The following theorem is the desired characterization of total paracompactness in real GO-spaces.

THEOREM 2.3. Given a GO-space $(\mathbf{R}, \tau)=\mathrm{GO}(R, E, I, L)$ on the real line, the following conditions are equivalent.

(a) $(\mathbf{R}, \tau)$ is totally paracompact.

(b) $(\mathbf{R}, \tau)$ is totally metacompact.

(c) $(\mathbf{R}, \tau)$ contains no DCE.

(d) For every nonempty real closed subspace $C$ there is a $\rho$-open subset $G$ such that $G \cap C \neq \varnothing$, and $\tau \mid(G \cap C)$ is either the discrete topology or the $\rho$-topology on $G \cap C$.

(e) There is a countable ordinal $\alpha$ and a $\rho$-open cover $\mathcal{G}=\left\{G_{\beta}: \beta<\alpha\right\}$ of $\mathbf{R}$ such that for each $\beta<\alpha, \tau$ restricted to the subspace $M_{\beta}=G_{\beta}-\bigcup\left\{G_{\gamma} \mid \gamma<\beta\right\}$ is either the discrete topology or the $\rho$-topology on $M_{\beta}$.

ProOF. (a) $\Rightarrow$ (b). Trivial.

(b) $\Rightarrow(\mathrm{c})$. This is the content of Theorem 1.7.

$(c) \Rightarrow(d)$. Suppose that $(\mathbf{R}, \tau)$ contains no DCE and let $C$ be a nonempty $\rho$-closed subset of $\mathbf{R}$. If $C$ contains a $\rho$-isolated point, we are done, so we may assume that $C$ is dense-in-itself. By Proposition 1.2, $r(C)$ and $l(C)$ are nowhere dense in the $\rho$-subspace topology of $C$. Then there are two cases.

Case 1. $C-i(C)$ is not dense in the $\rho$-topology of $C$. Then there is a $\rho$-open subset $G$ such that $\varnothing \neq G \cap C \subseteq i(C)$, that is, $\tau \mid(G \cap C)$ is the discrete topology.

Case 2. $C-i(C)$ is dense in the $\rho$-topology on $C$. Then, to avoid a DCE, $i(C)$ must be $\rho$-nowhere dense. Then

$$
r(C) \cup l(C) \cup i(C) \subseteq C-e(C)
$$

is nowhere dense in the $\rho$-topology of $C$, so there is a $\rho$-open set $G$ with $\varnothing \neq$ $G \cap C \subseteq e(C)$. Then $\tau \mid(G \cap C)$ is the $\rho$-topology.

$(\mathrm{d}) \Rightarrow(\mathrm{e})$. Obvious.

(e) $\Rightarrow$ (a). For a point $x \in \mathbf{R}$, let the rank $o(x)$ of $x$ be the unique ordinal $\beta$ with $x \in M_{\beta}$. By induction on $o(x)$ we shall prove that each $x$ has a totally paracompact $\tau$-neighborhood. Then the total paracompactness of $(\mathbf{R}, \tau)$ follows from Lemma 2.1. Suppose $\beta<\alpha$ and we are done for points with rank less than $\beta$. Then let $x \in M_{\beta}$ and let $G$ be a $\rho$-open set with $x \in G$ and $\operatorname{cl}(G) \subseteq G_{\beta}$. There are two cases to consider. 
Case 1. If $\tau \mid M_{\beta}$ is the discrete topology, then there is a $\tau$-open set $V$ with $V \subseteq G$ and $V \cap M_{\beta}=\{x\}$. By the induction hypothesis, each $y \in V-\{x\}$ has a totally paracompact $\tau$-neighborhood. Then, by Lemma $2.2, V$ is totally paracompact.

Case 2. If $\tau \mid M_{\beta}$ is the $\rho$-topology, then $C_{\beta}=\operatorname{cl}(G)-\bigcup\left\{G_{\gamma} \mid \gamma<\beta\right\}$ is compact in $\tau$ and by the induction hypothesis, each point in $\operatorname{cl}(G) \cap\left(\bigcup\left\{G_{\gamma} \mid \gamma<\beta\right\}\right)$ has a totally paracompact $\tau$-neighborhood. Then by Lemma $2.2, \operatorname{cl}(G)$ is a totally paracompact $\tau$-neighborhood of $x$.

COROLLARY 2.4. If a real GO-space $(\mathbf{R}, \tau)=\mathrm{GO}(R, E, I, L)$ is totally metacompact, then $(\mathbf{R}, \tau)$ is metrizable.

Proof. By Faber's Theorem [Fa, p. 48] it is enough to prove that $(\mathbf{R}, \tau)$ has a $\sigma$-closed-discrete dense subset $D \supseteq R \supseteq L$. Making use of 2.3(e), let

$$
A=\bigcup\left\{M_{\beta}|\tau| M_{\beta} \text { is discrete }\right\}
$$

and

$$
B=\bigcup\left\{M_{\beta}|\tau| M_{\beta} \text { is the } \rho \text {-topology }\right\} .
$$

Since each $M_{\beta}$ is an $F_{\sigma}$ subset in the $\rho$-topology, it follows that $A$ is $\sigma$-closed discrete in $(\mathbf{R}, \tau)$. Further, let $S$ be a countable $\rho$-dense subset of $(B, \tau \mid B)$. Since by Proposition 1.2, $R \cup L$ is countable, $D=A \cup S \cup R \cup L$ satisfies the conditions of Faber's theorem and, thus, $(\mathbf{R}, \tau)$ is metrizable.

The converse of Corollary 2.4 does not hold true. Indeed, the "anti-Michael line" $\mathrm{GO}(\varnothing, \mathbf{R}-\mathbf{Q}, \mathbf{Q}, \varnothing)$ is metrizable, but it is not totally metacompact because $[0,1]$ is a DCE in the subspace topology.

3. Subspaces of the real line. The following natural problem remains open.

PROBLEM 3.1. Suppose that $X$ is a subspace of a real GO-space. What is a necessary and sufficient condition for $X$ to be totally paracompact (or totally metacompact)?

Surprisingly enough we do not know the answer for subspaces of the real line with the usual topology.

PROBLEM 3.2. What subspaces of the real line are totally paracompact (or totally metacompact)?

The following partial results indicate some of the difficulties that arise when trying to answer the above problems.

THEOREM $3.3(\mathrm{MA}(\kappa))$. If $X$ is a second countable space which is the union of $\leq \kappa$ compact subsets, then $X$ is totally paracompact.

ProOF. Let $B$ be any base for $X$. Without loss of generality we may assume that $B$ is countable and closed under finite unions.

Let the poset $P$ consist of all finite functions from $\omega$ into $B \times B$ such that if $p(i)=\left\langle p_{1}(i), p_{2}(i)\right\rangle(i \in \operatorname{dom}(p))$, then for every $i \in \operatorname{dom}(p)$

(a) $\operatorname{cl}\left(p_{1}(i)\right) \subseteq \bigcup\left\{p_{2}(j) \mid j \in \operatorname{dom}(p), j \leq i\right\}$ and

(b) $p_{1}(i) \cap\left(\bigcup\left\{p_{2}(j): j \in \operatorname{dom}(p), j>i\right\}\right)=\varnothing$ hold. If $p$ and $q$ are in $P$, then define $p \leq q$ by $p \supseteq q$. Clearly, $|P| \leq \omega$ and thus $P$ is c.c.c. 
Claim. If $C$ is a compact subset of $X$, then $D(C)=\left\{p \in P: \bigcup\left\{p_{1}(i): i \in\right.\right.$ $\operatorname{dom}(p)\} \supseteq C$ \} is dense in $P$.

Indeed, given a $p \in P$, let

$$
C^{\prime}=C-\bigcup\left\{p_{2}(i): i \in \operatorname{dom}(p)\right\}
$$

Then let $n$ be bigger than the maximal element of $\operatorname{dom}(p)$ and define $p_{2}(n) \in B$ so that $p_{2}(n) \supseteq C^{\prime}$ and $p_{2}(n) \cap\left(\bigcup\left\{p_{1}(i): i \in \operatorname{dom}(p)\right\}\right)=\varnothing$. This can be done since $C^{\prime}$ is compact, $B$ is closed under finite unions and by (a),

$$
\bigcup\left\{\operatorname{cl}\left(p_{1}(i)\right): i \in \operatorname{dom}(p)\right\} \subseteq \bigcup\left\{p_{2}(i): i \in \operatorname{dom}(p)\right\} .
$$

Further define $p_{1}(n) \in B$ so that

$$
C \subseteq p_{1}(n) \subseteq \operatorname{cl}\left(p_{1}(n)\right) \subseteq \bigcup\left\{p_{2}(i): i \in \operatorname{dom}(p) \text { or } i=n\right\} .
$$

Then

$$
p \cup\left\{\left\langle p_{1}(n), p_{2}(n)\right\rangle\right\} \in D(C) .
$$

Let $X=\left\{C_{\alpha}: \alpha<\kappa\right\}$ be a cover of $X$ by compact subsets. By $\operatorname{MA}(\kappa)$, there is a filter $G \subseteq P$ such that $G$ meets each $D\left(C_{\alpha}\right)$. It is then easy to see that

$$
B^{\prime}=\left\{B \in B: \text { there is a } p \in G \text { and } i \in \omega \text { with } B=p_{2}(i)\right\}
$$

is a locally finite subcover of $B$.

COROLLARY 3.4. (a) Every $\sigma$-compact, second countable space is totally paracompact. (b) $(M A)$ Every subset of cardinality $<2^{\omega}$ of the real line is totally paracompact.

D. Lutzer asked (oral communication) whether a totally paracompact GO-space $X$ with a $G_{\delta}$-diagonal has to be metrizable. By Corollary 2.4, this is the case if $X$ is a GO-space on the real line.

ACKNOWLEDGMENT. Between the submission and acceptance of this paper the authors were informed that J. M. O'Farrell $\left[\mathbf{O F}_{\mathbf{2}}\right]$ independently obtained a generalization of Theorem 3.3.

\section{REFERENCES}

[BL 1 H. R. Bennett and D. J. Lutzer, eds., Topology and ordered structures, Part 1, Mathematical Centre Tracts No. 142, Amsterdam, 1981.

[BL $\mathbf{B L}_{2}$ H. R. Bennett and D. J. Lutzer, eds., Topology and ordered structures, Part 2, Mathematical Centre Tracts No. 169, Amsterdam, 1983.

[Fa] M. F. Faber, Metrizability in generalized ordered spaces, Mathematical Centre Tracts No. 53, Amsterdam, 1974.

[Fo] R. M. Ford, Basis properties in dimension theory, Doctoral Dissertation, Auburn University, Auburn, Ala., 1963.

[H] R. W. Heath, Screenability, pointwise paracompactness, and metrization of Moore spaces, Canad. J. Math. 16 (1964), 763-770.

[Le] A. Lelek, Mathematical Problem Book, Univ. of Houston, Problem No. 99.

[Lu] D. J. Lutzer, On generalized ordered spaces, Dissertationes Math. 89 (1971).

[M] E. Michael, The product of a normal space and a metric space need not be normal, Bull. Amer. Math. Soc. 61 (1963), 375-376.

[OF 1 J. M. O'Farrell, The Sorgenfrey Line is not totally metacompact, Houston J. Math 9 (1983), 271-273. 
[OF 2 ] J. M. O'Farrell, Some results concerning the Hurewicz property, Fund. Math. (to appear).

[S] R. Sorgenfrey, On the topological product of paracompact spaces, Bull. Amer. Math. Soc. 53 (1947).

[T] R. Telgarsky, Total paracompactness and paracompact dispersed spaces, Bull. Acad. Polon. Sci. Ser. Sci. Math. Astronom. 16 (1968), 567-572.

[TK] R. Telgarsky and H. Kok, The space of rationals is not absolutely paracompact, Fund. Math. 73 (1971), 75-78.

KosSUTH UNIVERSITY, DEBRECEN, H-4010, HUNGARY

Department of Mathematics, Texas Tech University, Lubbock, Texas 79409 